\title{
Evaluation of Hybrid PET/MRI for Gross Tumor Volume (GTV) Delineation in Colorectal Cancer Liver Metastases Radiotherapy
}

\author{
Yan-Nan Zhang ${ }^{1}, *$ \\ Xin Lu' ${ }^{1} *$ \\ Zhen-Guo Lu' \\ Li-Ping Fu' \\ Jun Zhao ${ }^{2}$ \\ Zuo-Lin Xiang'
}

'Department of Radiation Oncology, Shanghai East Hospital, School of

Medicine, Tongji University, Shanghai,

People's Republic of China; ${ }^{2}$ Department

of Nuclear Medicine, Shanghai East

Hospital, School of Medicine, Tongji

University, Shanghai, People's Republic of

China

*These authors contributed equally to this work
Correspondence: Zuo-Lin Xiang Department of Radiation Oncology, Shanghai East Hospital, School of Medicine, Tongji University, Shanghai, People's Republic of China Tel +86 02I-388045I8-I534I

Email xiangzuolinmd@hotmail.com
Purpose: Hybrid PET/MRI has been increasingly incorporated into the practice of radiation oncologists since it contains both anatomical and biological data and may bring about personalized radiation plans for each patient. The objective of this study was to evaluate the feasibility of GTV delineation from hybrid PET/MRI compared with that from currentpractice MRI during radiotherapy planning in patients with colorectal liver metastases.

Patients and Methods: Twenty-four patients (thirty lesions) with colorectal liver metastases were prospectively enrolled in this study. Three physicians delineated the target volume with the most popular delineating methods - the visual method. First of all, differences among the three observers were assessed. The difference and correlation of GTV values obtained by MRI, PET, and hybrid PET/MRI were subjected to statistical analysis afterwards. Finally, the dice similarity coefficient (DSC) was calculated to assess the spatial overlap. Based on the value of DSC, we also evaluate the correlation between DSC and tumor size. GTV-MRI was set as a reference.

Results: There was no significant difference among observers in GTV-MRI $(F=0.118$, $p=0.889)$, GTV-PET $(F=0.070, p=0.933)$ and GTV-PET/MRI $(F=0.40, p=0.961) .83 .33 \%$ of GTV-PET/MRI and $63.33 \%$ of GTV-PET were larger than the reference GTV-MRI. Statistical analysis revealed that GTV-PET/MRI $(p<0.001)$ and GTV-PET $(p<0.05)$ diverged statistically significantly from GTV-MRI. GTV-PET $(r=0.992, p<0.001)$ and GTV-PET/MRI $(r=0.997, p<0.001)$ were significantly related to GTV-MRI. The average DSC value between GTV-MRI and GTV-PET was 0.51 (range 0-0.90) and that between GTV-MRI and GTVPET/MRI was 0.72 (range $0.42-0.90$ ). There was a positive correlation between the DSC and GTV-MRI $(r=0.851, p<0.05)$.

Conclusion: With the database used, there is good agreement among observers. Hybrid PET/MRI in colorectal liver metastases radiotherapy may affect the GTV delineation. Moreover, the overlap degree between GTV-MRI and GTV-PET/MRI is higher and increases with volume.

Keywords: colorectal liver metastases, hybrid PET/MRI, radiotherapy, gross tumor volume

\section{Introduction}

The liver receives a double blood supply from the hepatic artery and portal vein, which makes the liver a likely site for the growth of metastatic tumors. Studies have shown that $25-50 \%$ of primary tumors can metastasize to the liver during their progression. ${ }^{1}$ In addition to regional lymph node metastases, liver is the second most prone to metastatic disease, often from primary colorectal, breast, lung, kidney, and skin cancers (melanoma). ${ }^{2}$ Metastasectomy is a radical treatment for 
liver metastases from colorectal cancer. Only a small number of patients initially undergo surgery, while for patients with inoperable colorectal liver metastases, stereotactic body radiation therapy (SBRT) has emerged as an effective, non-invasive alternative therapy. ${ }^{3}$ The current national comprehensive cancer network (NCCN) guidelines for colon and rectal cancer support aggressive local treatment of metastatic sites. ${ }^{4,5}$ SBRT characterized by high-accuracy positioning and dose delivery to the lesions and minimal exposure to normal tissues, has been applied in clinical practice. For liver metastases from colorectal cancer, the damage of SBRT to normal liver tissue is far less than that of traditional radiotherapy, the incidence of radiation-induced liver disease (RILD) treated with SBRT is also significantly reduced and multiple studies reported excellent local control. ${ }^{6,7}$ It is crucial to define the target volumes accurately for colorectal liver metastases SBRT, which can lead to fewer side effects or better outcomes with more precise target volumes.

At present, computed tomography (CT) and magnetic resonance imaging (MRI) are the most common imaging modalities for detecting and characterizing colorectal cancer liver metastases, and are widely used in target volume delineation. CT or MRI alone can show the morphologic features of the tumor but not the metabolic activity, while ${ }^{18} \mathrm{~F}$-fluorodeoxyglucose positron emission tomography $\left({ }^{18} \mathrm{~F}-\mathrm{FDG}\right.$ PET) images can reflect the metabolic activity of the tumor. In addition to its widespread use in the diagnoses and therapy monitoring of colorectal cancer patients with liver metastases, ${ }^{8,9}{ }^{18}$ F-FDG PET has recently increasingly been used in the delineation of target volumes for radiotherapy. The major disadvantages of PET/CT are the relatively high radiation dose from the two components and the relatively low spatial resolution of PET data. Compared with CT, MRI is more sensitive and accurate in detecting small liver lesions, which makes it an alternative to $\mathrm{CT}$ in hybrid imaging. ${ }^{10,11}$

In recent years, integrated PET/MRI technology has been put into clinical practice. The hybrid PET/MRI system, which combines the metabolic imaging function of PET with the excellent soft-tissue contrast of MRI, can provide more clinically relevant information than PET/ CT. ${ }^{11}$ It has a great effect on the precise delineation of the target volume in radiotherapy planning. To our knowledge, there is no report on the target volume delineation of PET/MRI in colorectal liver metastases radiotherapy planning. As a consequence, we put forward the hypothesis that PET/MRI could improve delineating precision in this situation. The aim of the study was to 1) assess the differences among different observers; 2) evaluate GTVs' size and spatial overlap to clarify the role of PET/MRI to be used to contour GTV.

\section{Materials and Methods Patients}

All of the patients we recruited were over 18 years old. Patients with other metastases, anaphylactic reaction upon receiving intravenous contrast medium or ${ }^{18} \mathrm{~F}-\mathrm{FDG}$, claustrophobia, metal device implantation or an inability to cooperate were excluded. In the end, 24 patients with colorectal liver metastases were prospectively enrolled and underwent PET/MRI scans prior to treatment between June 2019 and December 2020. This study was carried out in accordance with the recommendations of the local ethics committee of our hospital. Written informed consent was obtained from all participants.

\section{PET/MRI Image Acquisition}

The hybrid PET/MRI images were acquired in 3D mode. All patients received an intravenous injection of ${ }^{18} \mathrm{~F}-\mathrm{FDG}$ $(0.8-1.0 \mathrm{mCi} / \mathrm{kg}) \quad 50-60 \mathrm{~min}$ before PET image acquisition. In addition, a six-hour fast and bladder emptying and normoglycemic control were required prior to PET scanning. To minimize the impact of breathing movements on the boundary expansion, we used respiratory gating technology during the scan and the patients were adequately trained in respiratory movements prior to examination. During the process of reconstruction, the PET component of the system adopts Time of Flight (TOF) technology to display the image, which can reduce noise and provide higher sensitivity, thus increasing the image quality. The MRI built into the hybrid system is able to generate a magnetic field with a field strength of 3T.

The PET image was reconstructed by the ordered subsets expectation maximization (OSEM) algorithm (including 2 iterations, 20 subsets, $4 \mathrm{~mm}$ full width at half maximum (FWHM) Gaussian filter, and a 150×150 image matrix).

The diagnostic MRI scanning included T1-weighted high-resolution isotropic volume acquisition and T2weighted (T2W) 3D volume fast spin echo (FSE) imaging, both in the axial, sagittal and coronal orientations. T1 sequence parameters: repetition time (TR)/echo time $(\mathrm{TE})=5.04 / 2.24 \mathrm{~ms}, 4 \mathrm{~mm}$ slice thickness, $20 \%$ interslice gap, $350 \mathrm{~mm} \times 350 \mathrm{~mm}$ field of view (FOV), and a $256 \times 256$ matrix. Ax FSE T2 sequence parameters: TR/TE=3998/ 
$88.74 \mathrm{~ms}$, 6mm slice thickness, $20 \%$ interslice gap, $300 \mathrm{~mm} \times 300 \mathrm{~mm}$ FOV, and a $320 \times 320$ matrix.

\section{Tumor Volume Delineation}

All diagnostic MRI, PET, and hybrid PET/MRI images were confirmed by 2 radiologists that one works in the radiology department and the other in the nuclear medicine department, both of whom were board-certified and experienced in their disciplines. Three experienced radiation oncologists (A, B and C) performed the tumor delineations independently; lymph nodes were not delineated in our work. Thus, three GTVs (GTV-MRI, GTV-PET and GTV-PET/MRI) were defined by each of the three observers for each patient.

The GTV-MRI was manually contoured by radiation oncologists on axial T2-weighted MRI, with agreement by radiologists of radiology. On the basis of the patients' clinical history and related imaging examination (including MRI, CT and ultrasound), we considered that changes in mass, and nodule were abnormal and indicative of lesions. The images and reports of the MRI were blinded.

Until now, manual delineation of ${ }^{18}$ F-FDG PET-positive tissues has been the gold standard, despite poor reproducibility. ${ }^{12}$ After the radiation oncologists reviewed the PET images and the hybrid PET/MRI images with the radiologist of nuclear medicine, PET-GTV and GTV-PET /MRI were contoured. The GTV-PET/MRI was manually contoured on fusion of the PET images with the T2W axial images. The uptake of ${ }^{18}$ F-FDG significantly exceeded the normal surrounding tissue background, which we considered metabolic abnormalities and indicative of a lesion (Figure 1).

\section{Analysis}

Firstly, differences among the three observers were assessed. Meanwhile, GTVs obtained by PET and hybrid
PET/MRI were compared with the reference GTV-MRI. The average and median were calculated for obtained results. In addition, spatial analysis within GTV-MRI, GTV-PET and GTV-PET/MRI was performed. The DSC was calculated using the equation: $2 \times(\mathrm{A} \cap \mathrm{B}) /(\mathrm{A}+\mathrm{B})$, where $A$ and $B$ represent two volumes, $(A \cap B)$ represents the volume of the intersection, and $(\mathrm{A}+\mathrm{B})$ represents the absolute sum of their volumes. ${ }^{13}$ Subsequently, based on the value of DSC, we also evaluate the correlation between DSC (GTV-MRI vs GTV-PET/MRI) and tumor size.

All statistical analyses were carried out using IBM SPSS Statistics for Windows, Version 24.0 (IBM Corp., Armonk, NY, USA). The results of all quantitative data are expressed as the mean (SD). One-way analysis of variance (ANOVA) was used to compare the difference among the observers. A nonparametric paired-sample Wilcoxon signed-rank test was used to compare GTVs volumes based on MRI vs PET and MRI vs PET/MRI. Pearson analysis was used to calculate the correlation between GTV-MRI and GTV-PET, GTV-MRI and GTV-PET/MRI, DSC and the reference GTV-MRI. A result was considered statistically significant if the determined $\mathrm{p}$ value was less than $0.05(p<0.05)$.

\section{Results}

In our study, a total of 24 patients (30 lesions) were included. Of the 24 enrolled patients, nine had colon cancer liver metastases, fifteen had rectal cancer liver metastases, nine were female and fifteen were male. The median age of the patients was 61 years (range 35-85). The patients' characteristics are displayed in Table 1.

The GTV-MRI, GTV-PET, GTV-PET/MRI delineated by three observers were displayed in Table 2 . There was no significant difference among observers in tumor volume. The MRI-based mean (SD) GTV for all patients

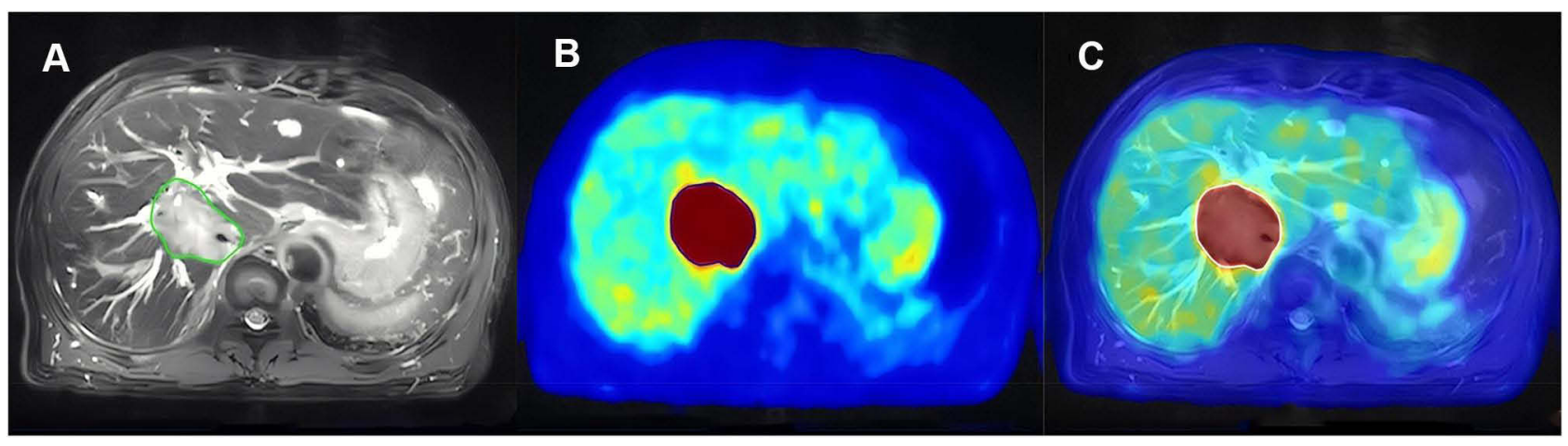

Figure I 73-year-old male with colorectal liver metastases. (A) The green line represents GTV-MRI; (B) The blue line represents GTV-PET; (C) The white line represents GTV-PET/MRI. 
Table I Patients Characteristics and Volume Results

\begin{tabular}{|l|c|}
\hline Characteristics & Value \\
\hline Age & $60(13)$ \\
Mean (SD) & $61(35-85)$ \\
Median (range) & \\
\hline Primary tumors & $15(62.5)$ \\
Rectal cancer, $\mathrm{n}(\%)$ & $9(37.5)$ \\
Colon cancer, $\mathrm{n}(\%)$ & \\
\hline GTV-MRI, cm & \\
Mean (SD) & $17.32(18.13)$ \\
Median (range) & $7.56(1.05-57.33)$ \\
\hline GTV-PET, cm ${ }^{3}$ & \\
Mean (SD) & $19.06(18.97)$ \\
Median (range) & $10.70(0.40-65.50)$ \\
\hline GTV-PET/MRI, cm ${ }^{3}$ & \\
Mean (SD) & $20.06(20.61)$ \\
Median (range) & $9.03(0.72-73.70)$ \\
\hline
\end{tabular}

Abbreviations: GTV, gross tumor volume; MRI, magnetic resonance imaging; PET, positron emission tomography.

Table 2 GTVs by Three Observers

\begin{tabular}{|l|l|l|l|}
\hline Observers & $\begin{array}{l}\text { GTV-MRI } \\
\left(\mathbf{c m}^{3}\right)\end{array}$ & $\begin{array}{l}\text { GTV-PET } \\
\left(\mathbf{c m}^{3}\right)\end{array}$ & $\begin{array}{l}\text { GTV-PET/MRI } \\
\left(\mathbf{c m}^{3}\right)\end{array}$ \\
\hline A & $16.22(17.03)$ & $18.51(19.41)$ & $18.75(18.17)$ \\
B & $16.39(17.51)$ & $17.87(18.80)$ & $19.13(19.71)$ \\
\hline & $19.34(20.21)$ & $21.81(21.73)$ & $21.20(21.73)$ \\
\hline & $F=0.274 p=0.761$ & $F=0.335 p=0.717$ & $F=0.131 p=0.877$ \\
\hline
\end{tabular}

Note: Data are reported as mean (SD).

Abbreviations: GTV, gross tumor volume; MRI, magnetic resonance imaging; PET, positron emission tomography.

was $17.32(18.13) \mathrm{cm}^{3}$. The PET-based mean (SD) GTV was 19.06 (18.97) $\mathrm{cm}^{3}$. The hybrid PET/MRI-based mean (SD) GTV was 20.06 (20.61) $\mathrm{cm}^{3} .86 .67 \%$ of GTV-PET /MRI and $66.67 \%$ of GTV-PET were larger than the reference GTV-MRI. Statistical analysis showed that GTV-PET /MRI $(p<0.001)$, GTV-PET $(p<0.05)$ diverged statistically significantly from the referenced GTV-MRI results (Figure 2). GTV-PET $(r=0.983, p<0.001)$ and GTV-PET /MRI $(r=0.992, p<0.001)$ were significantly related to reference GTV-MRI.

The average value of DSC between GTV-MRI and GTVPET was 0.52 (range 0.02-0.83) and between GTV-MRI and GTV-PET/MRI was 0.72 (range 0.42-0.89). Since the latter was greater than 0.70 , we chose the DSC between GTVMRI and GTV-PET/MRI to be assessed the correlation with the reference GTV-MRI ( $r=0.851, p<0.05$; Figure 3$)$.

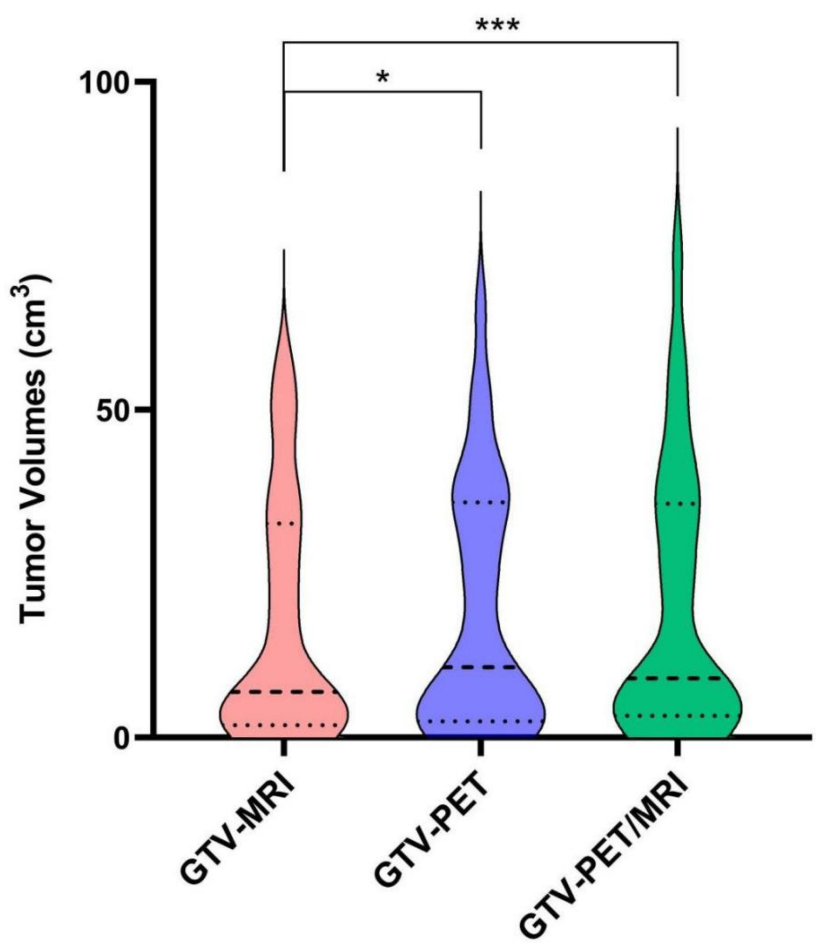

Figure 2 Violin plot of GTVs delineated based on MRI, PET and PET/MRI. The middle line shows the median values and the upper and lower whiskers indicate the range. Data are presented as the mean $\pm S D(n=30) .{ }^{*} p<0.05,{ }^{* * *} p<0.001$.

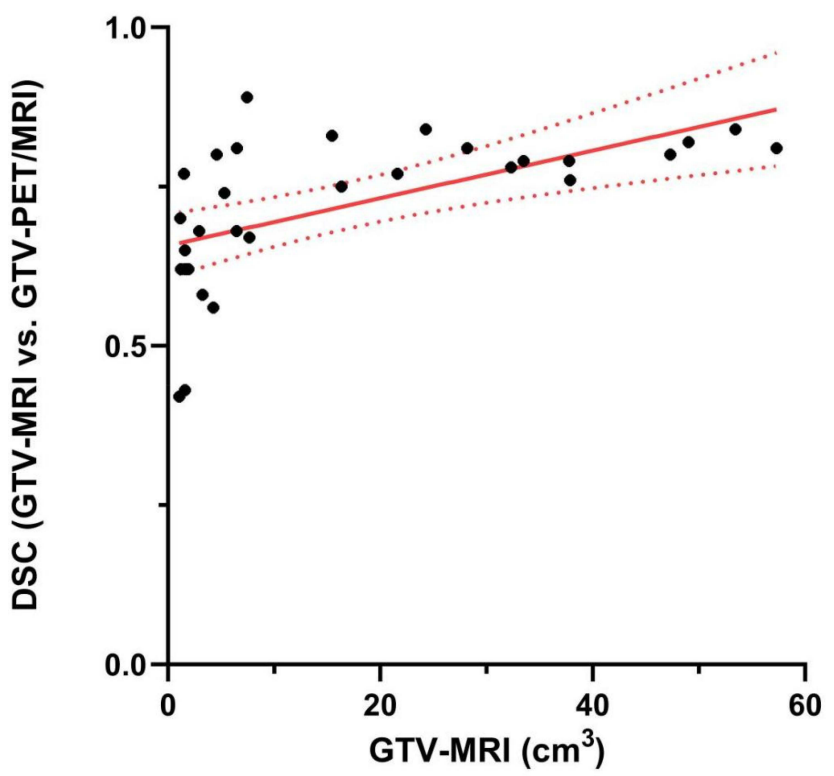

Figure 3 The correlation between GTV-MRI and DSC (GTV-MRI vs GTV-PET (MRI).

\section{Discussion}

The hybrid PET/MRI integrates the molecular metabolic information, morphology and function of the tumor, and can accurately distinguish the anatomical relationship 
between the tumor tissue and the surrounding adjacent tissue. At present, there is no consensus on the role of PET/MRI in delineating GTV, though various methods have been proposed and used to determine the contouring of ${ }^{18}$ F-FDG-positive tissue, which has led clinicians to reach different conclusions.

Currently, visual contour method - the radiologist identifies the hypermetabolic region of the tumor and determine the boundary visually to delineate GTV, is the most common method, which with lower technical requirements but highly depends on the observer. Therefore, three doctors with the qualification of attending physician or above were employed to identify the image, define the target area, and delineate the target volume. We compared the difference in the delineation of GTVs by three observers based on MRI, PET, and hybrid PET/MRI, respectively. The results (Table 2) showed that there was no significant difference among observers in tumor volume and it was concluded that the GTVs delineated had good consistency among them. And then, the average value was used for further analysis. We assessed the difference and correlation between target volumes delineated on MRI, PET, and PET/MRI. Our results showed that GTV-PET/MRI and GTV-PET were larger than GTV-MRI, GTV-PET/MRI $(p<0.001)$ and GTV-PET $(p<0.05)$ diverged statistically significantly from the referenced GTV-MRI. The reason may be that a microscopic tumor manifestation beyond the limits of the morphologic tumor volume is easily detectable with the PET component. In addition, hybrid PET/ MRI integrates the anatomical-, functional-, and molecular-level information of biological tissues, which can provide more clinical correlative information and higher accuracy. The true threshold of GTV needs to be validated using anatomical or pathological data, but it is challenging to obtain pathological confirmation because many patients with colorectal liver metastases have lost the opportunity for surgery, or some refused surgery. We speculate that the larger GTV may avoid the situation in which some lesions are not contoured in the target area and, therefore, are not irradiated, which may affect the efficacy of treatment. A large sample, multi-center, and prospective study is required to validate the hypothesis in the future.

The study on the target volume delineating with PET in colorectal liver metastases is rare. Bundschuh et $\mathrm{al}^{14}$ brought 18 patients with liver metastases (colon, breast and rectal cancer) into study and evaluate the role of respiratory-gated ${ }^{18}$ F-FDG PET/CT for target volume delineation in stereotactic radiation treatment of liver metastases. Their study showed that $13.8 \%$ GTV-PET is larger than GTV-MRI and found evidence for that ${ }^{18}$ F-FDG PET can improve the GTV delineation in liver metastases. Studies of other tumor locations (eg, esophagus, lung, pancreas, head and neck) have shown that the additional biological information of PET affects the variation in the GTV during radiation therapy, can reduce the risk of positioning error and influence the normal tissue dose-volume histogram and the calculation of probability values in corresponding normal tissue. ${ }^{15-18} \mathrm{Ma}$ et $\mathrm{al}^{18}$ showed that GTV contouring based on hybrid PET/MRI for head and neck cancers is feasible and may provide improved accuracy. In their study, GTV VIS and GTV FUS $_{\text {. }}$ were larger than GTV $\mathrm{GRI}_{\text {I }}$ with the use of hybrid PET/MRI images, which was similar to our findings, but tumor site determinations were different. Zhang et $\mathrm{al}^{19}$ reported that there were tumor volume discrepancies between GTVMRI and GTV-PET for cervical cancer. With the increase in tumor volume, the difference between GTV-MRI and GTV-PET increased. But some scholars have come to the opposite conclusion. Samolyk-Kogaczewska et $\mathrm{al}^{20}$ pointed out that the primary tumor volumes with manual delineation methods from MRI, PET and CT differed slightly from each other, but the differences were statistically irrelevant. While Wang et $\mathrm{al}^{13}$ demonstrated that hybrid PET/MRI- and CT-generated GTVs were similar overall and supplied similar radiation doses to the head and neck. The types of tumors differ from our study and the artifacts caused by tumor location, anatomical boundaries, clinical situations, and patient cooperation during examination have a great impact on the quality of the contouring. $^{21,22}$

Moreover, the spatial relationships appraisals were performed. The range of DSC is $0-1$. The value is 0 when there is no spatial overlap between imaging methodologies, while it is 1 when they completely overlap. Higher the DSC values indicate better coincidence degrees. Some scholars have noted that a DSC value $\geq 0.7$ indicates a better degree of overlap, ${ }^{23}$ suggesting that DSC may be highly correlated with the volume change. Our results show that the DSC value between GTV-MRI and GTV-PET/MRI - 0.72 - is higher, and the average DSC values were above 0.7 . The DSC value between GTV-PET and GTV-MRI was 0.52, lower than 0.7. In addition to the low spatial resolution of PET, another possible reason is the artifacts due to respiratory movement. During normal respiratory movement, organs can undergo displacements of up to $2 \mathrm{~cm},{ }^{24}$ and the liver is particularly vulnerable to respiratory motion, which may lead 
to the underestimation of lesion uptake and to the possibility of missing small lesions. Since the DSC between GTV-MRI and GTV-PET/MRI was greater than 0.70, we investigated the correlation with the reference GTV-MRI. There was a positive correlation between the DSC (GTV-MRI vs GTVPET/MRI $)$ and GTV-MRI $(r=0.851, p<0.05)$. The value of DSC is increased with the increase in tumor volume. It means as the tumor volume grows in size, the spatial overlap between GTV-MRI and GTV-PET/MRI became better. This finding is consistent with previous studies. ${ }^{25}$

The radiotherapy effects of target profiling based on PET/MRI are unknown. However, considering the statistically significant difference in the target volume, we have reason to suspect that this method has an impact on the therapeutic effect, which is of major importance for patients. All these findings inspire us to conduct some prospective research on the implementation of radiotherapy planning in future studies. Considering that colorectal liver metastases SBRT is a very precise treatment technique regarding the tissue exposed to radiation toxicity, any procedure designed to make radiotherapy planning more precise is of particular interest. Future studies on tumor volume delineation based on PET/MRI may benefit from more advanced technology such as MR-linac, novel radioactive tracers and respiratory-gated PET(4D-PET).

This study had a certain limitation. We included a relatively small number of patients. PET/MRI is an emerging diagnostic imaging technology, however, it is still relatively low in popularity at present due to its high price, metal contraindication during scanning and other reasons. In previous studies on PET/MRI-guided GTV delineation also included the small size of samples. ${ }^{13,18-20}$ For a more specific and accurate investigation of the advantages of PET/ MRI, a larger sample size study is required.

Taking into consideration our experience in colorectal liver metastases and studies of other cancers, we believe that delineation based on hybrid PET/MRI for colorectal liver metastases radiation therapy is worth considering. Further prospective studies in this and other settings are needed to assess the optimal use of PET/MRI for target volume contouring.

\section{Conclusion}

Target volume delineation based on hybrid PET/MRI requires establishing a clear methodology. With the database used, there is good agreement interobserver. Hybrid PET/MRI in colorectal liver metastases radiotherapy may affect the GTV delineation. In addition, we found that
GTV-PET/MRI and GTV-PET was larger than GTVMRI, which may allow adequate irradiation to the diseased tissue and better treatment effect. Moreover, the overlap degree between GTV-MRI and GTV-PET/MRI is higher and increases with volume. In the future, the role of PET/ MRI in radiation therapy cannot be underestimated, and its combination of biological and functional information provides an unprecedented opportunity for the development of radiotherapy towards more precise and personalized treatment planning.

\section{Abbreviations}

GTV, gross tumor volume; DSC, Dice similarity coefficient; SBRT, stereotactic body radiation therapy; NCCN, national comprehensive cancer network; RILD, radiationinduced liver disease; CT, computed tomography; MRI, magnetic resonance imaging; ${ }^{18} \mathrm{~F}-\mathrm{FDG}$ PET, ${ }^{18}$ F-fluorodeoxyglucose positron emission tomography; OSEM, ordered subsets expectation maximization; FWHM, full width at half maximum; T2W, T2-weighted; FSE, fast spin echo; TR, repetition time; TE, echo time; FOV, field of view; OAR, organ-at risk.

\section{Ethics Approval and Informed Consent}

The study protocol was approved by the institutional review board of Shanghai East Hospital. The study was conducted in accordance with the Declaration of Helsinki. Informed consent Written informed consent was obtained from all participants.

\section{Funding}

This work was supported by the National Natural Science Foundation of China (Grant No. 81960525), the Natural Science Foundation of Shanghai (Grant No. 17ZR1405300), the Science and Technology Supporting Project of Shanghai (Grant No.17411962600), the Science and the Technology Innovation Project of Shanghai (Grant No.19DZ1930900), the Pudong New Area Science and Technology Development Fund (Grant No. PKJ2018-Y02) and the Natural Science Foundation of Jiangxi (Grant No. 20192BAB205071).

\section{Disclosure}

The authors declare that there is no conflict of interest regarding the publication of this article. 


\section{References}

1. Koshariya M, Jagad RB, Kawamoto J, et al. An update and our experience with metastatic liver disease. Hepatogastroenterology. 2007;54(80):2232-2239.

2. Paley MR, Ros PR. Hepatic metastases. Radiol Clin North Am. 1998;36(2):349-363. doi:10.1016/S0033-8389(05)70027-0

3. Robin TP, Raben D, Schefter TE. A contemporary update on the role of stereotactic body radiation therapy (SBRT) for liver metastases in the evolving landscape of oligometastatic disease management Semin Radiat Oncol. 2018;28(4):288-294. doi:10.1016/j. semradonc.2018.06.009

4. National comprehensive cancer network $(\mathrm{NCCN})$ clinical practice guidelines in oncology. Colon Cancer. Version 2.2021. 2021.

5. National comprehensive cancer network $(\mathrm{NCCN})$ clinical practice guidelines in oncology. Rectal Cancer. Version 1.2021. 2021.

6. Petrelli F, Comito T, Barni S, Pancera G, Scorsetti M, Ghidini A. Stereotactic body radiotherapy for colorectal cancer liver metastases: a systematic review. Radiother Oncol. 2018;129(3):427-434. doi:10.1016/j.radonc.2018.06.035

7. Joo JH, Park JH, Kim JC, et al. Local control outcomes using stereotactic body radiation therapy for liver metastases from colorectal cancer. Int J Radiat Oncol Biol Phys. 2017;99(4):876-883. doi:10.1016/j.ijrobp.2017.07.030

8. Sacks A, Peller PJ, Surasi DS, Chatburn L, Mercier G, Subramaniam RM. Value of PET/CT in the management of liver metastases, part 1. AJR Am J Roentgenol. 2011;197(2):W256W259. doi:10.2214/AJR.10.6331

9. Brendle C, Schwenzer NF, Rempp H, et al. Assessment of metastatic colorectal cancer with hybrid imaging: comparison of reading performance using different combinations of anatomical and functional imaging techniques in PET/MRI and PET/CT in a short case series. Eur J Nucl Med Mol Imaging. 2016;43(1):123-132. doi:10.1007/ s00259-015-3137-z

10. Schulz A, Viktil E, Godt JC, et al. Diagnostic performance of CT, MRI and PET/CT in patients with suspected colorectal liver metastases: the superiority of MRI. Acta Radiol. 2016;57(9):1040-1048. doi: $10.1177 / 0284185115617349$

11. Riola-Parada C, García-Cãnamaque L, Pérez-Duẽnas V, GarcerantTafur M, Carreras-Delgado JL. Simultaneous PET/MRI vs. PET/CT in oncology. A systematic review. Rev Esp Med Nucl Imagen Mol. 2016;35(5):306-312. doi:10.1016/j.remn.2016.06.001

12. Devic S, Tomic N, Faria S, Menard S, Lisbona R, Lehnert S. Defining radiotherapy target volumes using $18 \mathrm{~F}$-fluoro-deoxyglucose positron emission tomography/computed tomography: still a Pandora's box? Int J Radiat Oncol Biol Phys. 2010;78 (5):1555-1562. doi:10.1016/j.ijrobp.2010.02.015

13. Wang K, Mullins BT, Falchook AD, et al. Evaluation of PET/MRI for tumor volume delineation for head and neck cancer. Front Oncol. 2017;7(8). doi:10.3389/fonc.2017.00008
14. Bundschuh RA, Andratschke N, Dinges J, et al. Respiratory gated [18F]FDG PET/CT for target volume delineation in stereotactic radiation treatment of liver metastases. Strahlenther Onkol. 2012;188(7):592-598. doi:10.1007/s00066-012-0094-3

15. Gondi V, Bradley K, Mehta M, et al. Impact of hybrid fluorodeoxyglucose positron-emission tomography/computed tomography on radiotherapy planning in esophageal and non-small-cell lung cancer. Int J Radiat Oncol Biol Phys. 2007;67(1):187-195. doi:10.1016/j. ijrobp.2006.09.033

16. Wilson JM, Mukherjee S, Chu KY, Brunner TB, Partridge M, Hawkins $\mathrm{M}$. Challenges in using ${ }^{18} \mathrm{~F}$-fluorodeoxyglucose-PET-CT to define a biological radiotherapy boost volume in locally advanced pancreatic cancer. Radiat Oncol. 2014;9:146. doi:10.1186/1748717X-9-146

17. Muijs CT, Schreurs LM, Busz DM, et al. Consequences of additional use of PET information for target volume delineation and radiotherapy dose distribution for esophageal cancer. Radiother Oncol. 2009;93(3):447-453. doi:10.1016/j.radonc.2009.08.030

18. Ma JT, Han CB, Zheng JH, et al. Hybrid PET/MRI-based delineation of gross tumor volume in head and neck cancer and tumor parameter analysis. Nucl Med Commun. 2017;38(7):642-649. doi:10.1097/ MNM.0000000000000687

19. Zhang SM, Xin J, Guo QY, Ma JT, Ma JT, Sun HZ. Comparison of tumor volume between PET and MRI in cervical cancer with hybrid PET/MR. Int J Gynecol Cancer. 2014;24(4):744-750. doi:10.1097/ IGC.0000000000000097

20. Samolyk-Kogaczewska N, Sierko E, Zuzda K, Gugnacki P, Szumowski P. PET/MRI-guided GTV delineation during radiotherapy planning in patients with squamous cell carcinoma of the tongue. Strahlenther Onkol. 2019;195(9):780-791. doi:10.1007/s00066-01901480-3

21. MacManus M, Nestle U, Rosenzweig KE, et al. Use of PET and PET/ CT for radiation therapy planning: IAEA expert report 2006-2007. Radiother Oncol. 2009;91(1):85-94. doi:10.1016/j. radonc.2008.11.008

22. Belli ML, Mori M, Broggi S, et al. Quantifying the robustness of [(18)F]FDG-PET/CT radiomic features with respect to tumor delineation in head and neck and pancreatic cancer patients. Phys Med. 2018;49:105-111. doi:10.1016/j.ejmp.2018.05.013

23. Zou KH, Warfield SK, Bharatha A, et al. Statistical validation of image segmentation quality based on a spatial overlap index. Acad Radiol. 2004;11(2):178-189. doi:10.1016/S1076-6332(03)00671-8

24. Riou O, Serrano B, Azria D, et al. Integrating respiratory-gated PET-based target volume delineation in liver SBRT planning, a pilot study. Radiat Oncol. 2014;9(1):127. doi:10.1186/1748-717X9-127

25. Ma DJ, Zhu JM, Grigsby PW. Tumor volume discrepancies between FDG-PET and MRI for cervical cancer. Radiother Oncol. 2011;98 (1):139-142. doi:10.1016/j.radonc.2010.10.004
Cancer Management and Research is an international, peer-reviewed open access journal focusing on cancer research and the optimal use of preventative and integrated treatment interventions to achieve improved outcomes, enhanced survival and quality of life for the cancer patient.
The manuscript management system is completely online and includes a very quick and fair peer-review system, which is all easy to use. Visit http://www.dovepress.com/testimonials.php to read real quotes from published authors. 